DOI: https://doi.org/10.46296/yc.v4i7.0049

\title{
LA GESTIÓN DE CALIDAD COMO ESTRATEGIA DE MEJORA DE LOS PROCESOS ADMINISTRATIVOS EN LA DISTRIBUIDORA TELMO MACÍAS MORA "T.M.M.".
}

\section{QUALITY MANAGEMENT AS A STRATEGY TO IMPROVE ADMINISTRATIVE PROCESSES AT DISTRIBUIDORA TELMO MACÍAS MORA "T.M.M.".}

\author{
Quijano-Macías Carlina Giovanna1*; Bravo-Giler María Amelia² \\ 1,2 Universidad Técnica de Manabí, UTM. Portoviejo, Ecuador. \\ *Correo: carlig125@hotmail.com
}

\begin{abstract}
Resumen
La meta del presente artículo fue establecer los procesos de la gestión de calidad como estrategia de mejora de los procesos administrativos en la Distribuidora Telmo Macías Mora "T.M.M." siendo esta uno de los principales instrumentos para el crecimiento de las empresas. Para el logro del objetivo del artículo (de tipo exploratorio y descriptivo), se ha hecho una amplia revisión de diversas fuentes documentales científicas, teóricas y estadísticas. Esta información ha sido extraída, analizada y correlacionada en su totalidad, proceso que viabilizo el desarrollo de un diagnostico situacional de la distribuidora, mediante la que es estableció la falta de Manual de Procedimientos y organización, la carencia de Políticas en la empresa, el no contar con misión y visión institucional, así como la falta de un organigrama formal hace evidente la necesidad de aplicar un proceso de mejora en base a un sistema de gestión.
\end{abstract}

Palabras clave: Gestión de calidad, mejora de los procesos, procesos administrativos.

\begin{abstract}
The goal of this article was to establish the processes of quality management as a strategy to improve administrative processes at Distribuidora Telmo Macías Mora "T.M.M." This being one of the main instruments for the growth of companies. In order to achieve the objective of the article (exploratory and descriptive), a broad review of various scientific, theoretical and statistical documentary sources has been made. This information has been extracted, analyzed and correlated in its entirety, a process that made possible the development of a situational diagnosis of the distributor, through which the lack of a Procedures Manual and organization, the lack of Policies in the company, the lack of With an institutional mission and vision, as well as the lack of a formal organization chart, it is evident the need to apply an improvement process based on a management system.
\end{abstract}

Keywords: Quality management, process improvement, administrative processes.

Información del manuscrito:

Fecha de recepción: 07 de mayo de 2020.

Fecha de aceptación: 09 de julio de 2020.

Fecha de publicación: 10 de julio de 2020 


\section{Introducción}

En la actualidad el contar con una adecuada Gestión Administrativa se ha convertido en un factor de gran importancia para todas las empresas, debido a que por medio de este es posible aplicar técnicas para tener un desarrollo más eficiente y eficaz.

Los autores Fergusson \& Langford, (2017) dan a conocer que: "Las organizaciones en el momento de ofrecer un servicio o producto deberían aplicar un Sistema de Gestión Administrativa debido a que les permite seguir un ciclo basado en los principios administrativos." (pág. 76). En la actualidad la calidad en las instituciones ya sean públicas o privadas, juega un rol esencial para su desarrollo, siendo así que, hoy en día, se habla de calidad del trabajo, calidad del proceso, calidad de sistemas, calidad de servicios, entre otros.

El mejoramiento de los procesos de gestión de calidad se ve determinado por la gestión administrativa, pues el contar con una institución adecuadamente estructurada, basada en un cimentado esquema organizacional, implicaría el crecimiento y fortalecimiento de esta. Caso contrario el no contar con una adecuada gestión administrativa genera que las instituciones presenten falencias en sus actividades, afectando su estructura orgánica, las metas institucionales.

Esta problemática es palpable a nivel internacional, generado una serie de estudios entorno a esta temática, con la finalidad de mejorar los procesos de calidad institucional. Partiendo de ello es posible citar al autor Riveros Ortiz, (2016) en su investigación "Propuesta para el mejoramiento del proceso de gestión administrativa de la empresa SYSLAN SAS" realizada en la Universidad Católica de Colombia en la que expresa:

Aunque la empresa lleva alrededor de 6 años en el mercado y que tiene un grupo importante de clientes, no se ha propuesto realizar una evaluación del servicio, ni existen políticas encaminadas a la atención del cliente en pro de la fidelización, recomendación y preferencia de los clientes. (pág. 49)

Para el autor del estudio, la falta de los procesos administrativos afecta directamente a los clientes, debido a 
que una institución que no esté adecamente cimentada en los procesos de gestión administrativa y de calidad no podrá brindar de manera oportuna los servicios que estos requieran.

A nivel nacional es posible citar el estudio llevado a cabo por Lazo Cordero \& Macas Moreno, (2018) titulado "Propuesta de mejoras en gestión administrativa en la empresa Modermueble S.A. Ciudad de Guayaquil", estudio que tuvo como finalidad "Mejorar la Gestión Administrativas en la empresa Modermueble S.A con el fin de elaborar e implementar un manual de funciones y procedimientos para simplificar los procesos administrativos", meta alcanzada por medio de la aplicación de estudio bibliográfico y de campo, mediante la cual se pudo concluir que:

El desarrollar un manual interno, permitirá que los empleados conozcan de mejor manera la empresa, el cómo se encuentra constituida, los procedimientos, políticas con las que cuenta y que debe ser respetada para disminuir errores operativos y evitar problemas en la organización. (pág. 97)

Se hace evidente que, en los procesos de gestión de calidad y gestión administrativa, se hace fundamental el desarrollo del manual organizacional, para que la empresa actúe de forma coordinada conforme a las metas institucionales.Para efectos del presente estudio se consideró un proceso de diagnóstico en la Distribuidora Telmo Macías Mora "T.M.M, esto a raíz de los problemas de organización y comercialización de la compañía nace la necesidad de establecer estrategias para mejorar su calidad de gestión administrativa. La empresa como toda compañía busca mejorar su sistema de estructuración organizacional para que les ayude a implementar una mejor organización y distribución de trabajo con la finalidad de mejorar el clima laboral y lograr que sus colaboradores sean un grupo humano motivado.

Partiendo de ello, el presente artículo tiene como finalidad establecer los procesos de la gestión de calidad como estrategia de mejora de los 
procesos administrativos en la Distribuidora Telmo Macías Mora "T.M.M.".

\section{Metodología}

\subsection{Modalidad de la investigación}

Investigación Cualitativa: Tiene como características comunes referirse a sucesos complejos que tratan de ser descritos en su totalidad, en su medio natural. "No hay consecuentemente, una abstracción de propiedades 0 variables para analizarlas mediante técnicas estadísticas apropiadas para su descripción y la determinación de correlaciones" (Rodríguez Gómez, 1996)

En efectos del presente estudio, la investigación cualitativa, permite la caracterización de las variables de estudio, tanto en su entorno practico, así como teórico. Meta que será lograda por medio de los instrumentos de recolección de información, así como los procesos de estudio de campo que se llevaran a cabo.

\subsection{Tipo y diseño de la investigación}

Investigación de campo: La investigación de campo o trabajo de campo es la recopilación de información fuera de un laboratorio o lugar de trabajo. Es decir, los datos que se necesitan para hacer la investigación se toman en ambientes reales no controlados.

Se seleccionó este tipo de investigación debido a que gran parte de las actividades de recopilación de información se realizaran en el campo directo de investigación, siendo la Distribuidora Telmo Macías Mora "T.M.M.".

Investigación Bibliográfica: La investigación bibliográfica constituye una excelente introducción a todos los otros tipos de investigación, además de que constituye una necesaria primera etapa de todas ellas, puesto que ésta proporciona el conocimiento de las investigaciones ya existentes -teorías, hipótesis, experimentos, resultados, instrumentos y técnicas usadasacerca del tema o problema que el investigador se propone investigar o resolver.

\subsection{Definición de la población y muestra.}


Para el presente estudio se consideró como población o universo al personal que labora en las áreas administrativas de la Distribuidora Telmo Macías Mora "T.M.M.", siendo un total de 40 personas; debido al tamaño de la población no se aplicó formula de muestra y se consideró la totalidad de esta.

\subsection{Métodos}

Deductiva - Inductiva: Método que se caracteriza por el análisis de lo general a lo particular; la aplicación de este método permitirá el estudio de la problemática de la investigación tanto a nivel internacional, nacional y local, basándose en el análisis de investigaciones previas, además que permitirá la generación de conclusiones específicas por medio del proceso de recolección de información de fuentes primarias y secundarias.

Análisis - síntesis: Una de las principales características de este método es que se refiere a dos actividades complementarias en lo que refiere al estudio de realidades complejas. La primera de estas dos actividades es el análisis, mismo que consiste en la separación de las partes de esas realidades hasta llegar a conocer sus elementos fundamentales y las relaciones adyacentes que se presentan entre las mismas.

La utilización del método de análisis síntesis permitió conocer de forma más profunda las variables que se estén estudiando en relación con su entorno de influencia, permitiendo simplificar su descripción, descubrir relaciones aparentemente ocultas y construir nuevos conocimientos a partir de otros que ya se posean.

\subsection{Técnicas e instrumentos de recolección de información}

Encuesta: Técnica investigativa aplicada para la recolección de información cuantificable y medible, se encuentra caracterizada por la aplicación de un instrumento con preguntas objetivas y cerradas. Esta técnica se aplicó con la población selecciona, que, para fines del estudio, corresponde al personal que labora en las áreas administrativas de la Distribuidora Telmo Macías Mora "T.M.M." 


\section{Resultados}

\subsection{Gestión de la Calidad.}

Abellan, (2015) determina que "la Gestión de la calidad se ha convertido actualmente en la condición necesaria para cualquier estrategia dirigida hacia el éxito competitivo de la empresa" (pág. 74). El aumento incesante del nivel de exigencia del consumidor, junto a la explosión de competencia de nuevos países comparativas en costos y la creciente complejidad de productos, procesos, sistemas y organizaciones son algunas las causas que hacen de la calidad un factor determinante para la competitividad y la supervivencia de la empresa moderna.

\subsection{Modelo de Gestión de la} Calidad.

Una de las consecuencias de la globalización de los mercados, es la exigencia de los clientes, quienes más conocedores y complejos, ahora saben elegir con decisión. Por su parte, los gerentes reconocen que se pueden obtener ventajas competitivas sustanciales mediante un mejor servicio al cliente, especificar por lo que el mismo se ha convertido en un medio poderoso para diferenciar una empresa de sus competidores.

Es por ello la importancia de implementar Modelos de Gestión de la Calidad (MGC) para que las empresas permanezcan en el mercado competitivo.

Lara Ocaña, (2017) sobre el modelo de gestión de calidad determina que consiste en reunir todas las actividades y funciones en forma tal que ninguna de ellas esté subordinada a las otras y que cada una se planee, controle y ejecute de un modo formal y sistemático.

Barragan, (2016) , da a conocer que los modelos de gestión de calidad, tiene como objetivo principal que las empresas desarrollen sistemáticamente, productos, bienes y servicios de mejor calidad y cumplan con las necesidades $y$ deseos de los clientes, así también una la misión de la empresa con el esfuerzo de cada área, en una sinergia de resultados hacia la competitividad y la calidad de clase mundial.

Es decir, un modelo de gestión de calidad con procesos y 
procedimientos ágiles y comprensibles para todos los involucrados, pasando por las etapas de diseño, materias primas, fabricación, distribución, entrega y satisfacción del cliente.

Cuando una empresa decide implantar un modelo de gestión de calidad, es señal de que la empresa tiene el propósito de permanecer y crecer como organización, ser competitiva, proteger los intereses de los accionistas, cuidar la fuente de trabajo y mejorar la calidad de vida de su personal.

Para tener éxito en la implantación de un Modelo de Gestión de Calidad Barreiro Cedeño, Delgado Chávez, Garcia Ponce, \& Mendoza Zamora, (2018) consideran necesario que los directivos comprendan la necesidad de fomentar los siguientes aspectos en la empresa:

- Establecer una cultura de calidad en la empresa.

- Establecer la atención centrada en el cliente creando el máximo valor.
- Inculcar en toda la premisa de hacerlo bien, a la primera vez y siempre.

- Crear constancia y ser perseverante con el propósito de mejorar los productos $y$ servicios.

- Realizar propuestas de innovación para mejorar la efectividad de la cadena de valor.

- Establecer que los procesos, los métodos y sistemas deben estar sujetos a ciclos de mejora continua.

- Establecer un programa para el diseño e implantación de los procesos y sistemas que integran el modelo de calidad.

- Contribuir con la sociedad promoviendo los valores de calidad y generando un compromiso con el bienestar de la sociedad y con la conservación del medio ambiente.

\subsection{Cultura organizacional}

Existen múltiples y diferentes definiciones sobre cultura organizacional, pero es posible 
establecerla como un sistema de creencias, valores, que se manifiestan en normas, actitudes, conductas, comportamientos, relaciones interpersonales, el estilo de liderazgo, que se desarrollan en un grupo dentro de la organización, o en una organización entendido como grupo, con el fin de dar cumplimiento a la materialización de la visión en su interacción con el entorno en un tiempo dado.

Dávalos Mayorga, (2016, pág. 112) establece que la cultura es propia de cada organización, y siempre hay una cultura. Es un proceso evolutivo donde sus elementos varían con el cursar del tiempo, y unos se extinguen y otros se solidifican, pero la cultura como tal no desaparece, sino que sufre procesos continuos de transformación"

Aleman Carmona, (2018, pág. 134) establece que la cultura organizacional ofrece formas definidas de pensamiento, sentimiento y reacción que guían la toma, a su vez incluye lineamientos perdurables mientras cumple las siguientes funciones:
- Transmitir un sentimiento de identidad a los miembros de la organización

- Facilitar el compromiso con algo mayor que el yo mismo

- Reforzar la estabilidad del sistema social

- Ofrecer premisas reconocidas y aceptadas para la toma decisiones

Los administradores eficientes hacen uso de símbolos, creencias y rituales de la cultura para lograr la aceptación de metas, motivar a la gente y asegurar su compromiso a largo plazo.

Coaguila Gonzales, (2017) detalla que hoy en día, es necesario que las organizaciones diseñen procesos y estructuras más flexibles al cambio, y que se produzca, no como un proceso forzado de imposición de normas procedimientos, sino como consecuencia del aprendizaje de sus integrantes.

Esto implica generar condiciones para promover equipos de alto desempeño, entendiendo que el aprendizaje en equipo implica generar valor al trabajo y más adaptabilidad al cambio con una amplia visión hacia la innovación. 
Han sido muchos los esfuerzos de transformación que han fracasado en las organizaciones por falta de planificación de este proceso y del liderazgo en esas organizaciones, lo cual ha generado el deterioro en sus funciones generales. Es por ello por lo que todo proceso de organización debe de estar acompañado por procesos que permitan la obtención de adecuados resultados institucionales.

\subsection{Gestión Administrativa.}

Los autores Ramírez Casco, Ramírez Garrido, \& Calderón Moran, (2017) señanalan que la gestión administrativa en una empresa es uno de los factores más importantes cuando se trata de emprender un negocio debido a que de ella dependerá el éxito que tenga dicho negocio o empresa planeamiento, la organización, la dirección y el control, desempeñada para determinar y lograr objetivos manifestados mediante el uso de seres humanos y de otros recursos.

En el sector privado uno de los principales propósitos de la gestión administrativa es proporcionar información para la toma de decisiones estratégicas.

Partiendo de este proceso en las instituciones se derivan muchas decisiones claves en la administración mismas que suelen estar basadas en los resultados futuros.

\subsection{Elementos de la Gestión} Administrativa

Mendoza Briones, (2017) define el acto de administrar como planeación, organización, dirección y controlar. Las funciones engloban los elementos de la administración. Estos mismos elementos constituyen el proceso administrativo que pueden ser encontrados en cualquier área de la empresa, es decir, que cada cual desempeña actividades de planeación, organización, dirección y control, como actividades administrativas esenciales. A continuación, se describe dichos elementos:

A. Planeación: Consiste en saber qué se va a hacer por anticipado, cuál va a ser la dirección que se va a tomar para alcanzar los objetivos de 
la manera más eficiente. ¿Qué se desea conseguir (objetivos)? ¿Qué se va a hacer para alcanzarlo? ¿Quién y cuándo lo va a hacer? ¿Cómo lo va a hacer (recursos)? La planeación trata de crear un futuro deseado.

Hernández \& Cardona, (2017) considera que esta etapa abarca la definición de las metas de la organización, el establecimiento de una estrategia general para alcanzar esas metas y el desarrollo de una jerarquía minuciosa de los planes para integrar y coordinar las actividades.

B. Organización: "La organización es un sistema que permite una utilización equilibrada de los recursos cuyo fin es establecer una relación entre el trabajo y el personal que lo debe ejecutar" (Coque Arboleda, 2016, pág. 45). Es un proceso en donde se determina qué se debe hacerse para lograr una finalidad establecida o planeada, dividiendo y coordinando las actividades y suministrando los recursos. Elementos de la organización:

División del trabajo: "Para dividir el trabajo es necesario seguir una secuencia que abarca las siguientes etapas: La primera; (jerarquización) que dispone de las funciones del grupo social por orden de rango, grado o importancia" (Pitchford, 2017). La segunda; (departamentalización) que divide y agrupa todas las funciones $y$ actividades, en unidades específicas, con base en su similitud.

Coordinación: "Es la sincronización de los recursos y los esfuerzos de un grupo social, con el fin de lograr oportunidad, unidad, armonía y rapidez, en el desarrollo de los objetivos" (Barragan 2016, 89).

Dirección: "La dirección es la función que trata, a través de la influencia interpersonal, de lograr que todos los involucrados en la organización contribuyan al logro de sus objetivos. Se ejerce a través de tres subfunciones: el liderazgo, la motivación y la comunicación" (Fergusson \& Langford, 2017, pág. 64).

D. Control: "El control es la función que efectúa la medición de los resultados obtenidos comparándolos con los esperados (planeados) con el fin de buscar la mejora continua". 
El control es la actividad de seguimiento encaminada a corregir las desviaciones que puedan darse respecto a los objetivos. El control se ejerce con referencia a los planes, mediante la comparación regular y sistemática de las previsiones y las consiguientes realizaciones y la valoración de las desviaciones habidas respecto de los objetivos. "El control, pues, contrasta lo planeado y lo conseguido para desencadenar las acciones correctoras, que mantengan el sistema regulado, es decir, orientado a sus objetivos."

\subsection{Diagnóstico aplicado en la} Distribuidora Telmo Macías Mora "T.M.M.".

La Distribuidora Telmo Macías Mora "T.M.M." con RUC: 1302604549001 inicio sus actividades el 1 enero/ 2000, teniendo como actividad comercial venta al por mayor de alimentos en general desde los inicios se ha dedicado a la distribución de productos de las siguientes líneas comerciales: Conservas Isabel, Industrias Ales, llegando a expandirse a las líneas de familia Sancela, PROALCO, MANZHI, DISPACIF con los que mantienen hasta la actualidad; entre los clientes principales de la institución se encuentran: Comisariatos Velboni, Comercial Casanova, Comisariato Gonzalo Zambrano.

A pesar de ya tener 20 años en el mercado se presentan problemas en los procesos de gestión administrativa, razón por la que se le aplico un proceso de diagnóstico como se detalla a continuación.

Tabla 1. Resultados del proceso de diagnóstico.

\begin{tabular}{|l|c|c|c|c|c|c|}
\hline \multicolumn{1}{|c|}{ Preguntas } & \multicolumn{2}{c|}{ Siempre } & \multicolumn{2}{c|}{$\begin{array}{c}\text { Algunas } \\
\text { veces }\end{array}$} & \multicolumn{2}{|c|}{ Nunca } \\
\cline { 2 - 8 } & $\mathrm{F}$ & $\%$ & $\mathrm{~F}$ & $\%$ & $\mathrm{~F}$ & $\%$ \\
\hline $\begin{array}{l}\text { 1. ¿La gerencia general de la institución transmite la visión, } \\
\text { misión y objetivos estratégicos? }\end{array}$ & 0 & 0 & 0 & 0 & 40 & 100 \\
\hline $\begin{array}{l}\text { 2. ¿El plan operativo de la institución es comunicado al } \\
\text { personal de forma oportuna? }\end{array}$ & 0 & 0 & 0 & 0 & 40 & 100 \\
\hline $\begin{array}{l}\text { 3. ¿La institución suele contar con un plan de formación y } \\
\text { capacitación que se comunique de forma oportuna? }\end{array}$ & 5 & 13 & 18 & 45 & 17 & 43 \\
\hline $\begin{array}{l}\text { 4. ¿Considera Ud. que la Alta Dirección desarrolla } \\
\text { controles sobre el presupuesto? }\end{array}$ & 23 & 58 & 8 & 20 & 9 & 23 \\
\hline $\begin{array}{l}\text { 5. ¿Considera Ud. que el presupuesto institucional } \\
\text { comprende las necesidades de la Institución? }\end{array}$ & 25 & 63 & 7 & 18 & 8 & 20 \\
\hline $\begin{array}{l}\text { 6. ¿Considera Ud. la estructura organizacional facilita la } \\
\text { comunicación entre los niveles de la institución? }\end{array}$ & 0 & 0 & 0 & 0 & 40 & 100 \\
\hline $\begin{array}{l}\text { 7. ¿Considera Ud. que la estructura organizacional } \\
\text { contribuye al cumplimiento de los objetivos institucionales? }\end{array}$ & 18 & 45 & 19 & 48 & 3 & 8 \\
\hline $\begin{array}{l}\text { 8. ¿Considera Ud. que la asignación y distribución de } \\
\text { cargos responde a los requerimientos? }\end{array}$ & 19 & 48 & 16 & 40 & 5 & 13 \\
\hline 9. ¿Los empleados son evaluados permanentemente? & 19 & 48 & 12 & 30 & 9 & 23 \\
\hline $\begin{array}{l}\text { 10. ¿Considera Ud. que existe una coordinación adecuada } \\
\text { entre las áreas administrativas? }\end{array}$ & 15 & 38 & 16 & 40 & 9 & 23 \\
\hline $\begin{array}{l}\text { 11. ¿Considera Ud. que los procesos administrativos } \\
\text { cuentan con controles que aseguran la eficacia? }\end{array}$ & 12 & 30 & 14 & 35 & 14 & 35 \\
\hline $\begin{array}{l}\text { 12. ¿Considera Ud. que el cumplimiento de las metas va de } \\
\text { acuerdo a lo programado por la institución? }\end{array}$ & 16 & 40 & 13 & 33 & 11 & 28 \\
\hline
\end{tabular}

Elaborado por: Autora

Fuente: Instrumento de recolección de información. 
Figura 1: Resultados del proceso de diagnóstico.

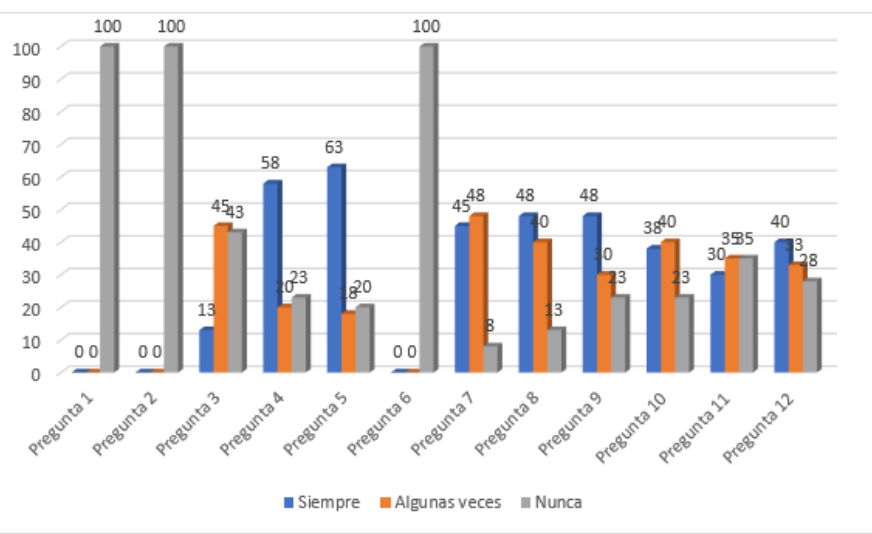

Elaborado por: Autora

Fuente: Instrumento de recolección de información.

Partiendo del proceso de diagnóstico aplicado al personal de la Distribuidora Telmo Macías Mora "T.M.M." es posible determinar los siguientes aspectos:

El $100 \%$ de los encuestados dio a conocer que la visión, misión y objetivos estratégicos no se transmiten de forma oportuna.

El $100 \%$ de los encuestados dio a conocer que el plan operativo de la institución no se transmite de forma oportuna.

El $13 \%$ de los encuestados respondió que siempre se suele contar con un plan de formación y capacitación el mismo que se comunica de forma oportuna, el $45 \%$ expreso que algunas veces se han llevado a cabo el plan de capacitación y el 43\% restante expreso que nunca se generan dichos procesos.

El 58\% dio a conocer que siempre se desarrollan controles sobre el presupuesto, el $20 \%$ dio a conocer que a veces se generan dichos controles y el $23 \%$ restante expreso que nunca se desarrollan estos controles de manera oportuna.

El $63 \%$ dio a conocer que el presupuesto institucional siempre se ajusta a las necesidades de la institución, el $18 \%$ dio a conocer que a veces el presupuesto se ajusta a las necesidades institucionales, el $20 \%$ expreso que nuca se ajusta a las necesidades de la institución, por lo que hay la necesidad de reajustarlos según se presente la necesidad.

El $100 \%$ de los encuestados dio a conocer que la institución no cuenta con una estructura organizacional que facilite la comunicación entre los niveles de la institución.

El $45 \%$ de los encuestados consideran que la estructura organizacional siempre contribuye al cumplimiento de los objetivos, el $48 \%$ dio a conocer que a veces y el 
$8 \%$ expreso que nunca, a pesar de no contar con un organigrama institucional, la institución cuenta con una adecuada estructura orgánica y una correcta distribución de funciones.

El $48 \%$ de los encuestados consideran que la asignación y distribución de cargos siempre responde a los requerimientos, el $40 \%$ dio a conocer que a veces y el $13 \%$ expreso que nunca.

El $48 \%$ de los encuestados respondió que los empleados siempre son evaluados permanentemente, el $30 \%$ expreso que a veces de generan procesos de evaluación y el $23 \%$ determino que la distribuidora no genera procesos de evaluación interna

El $38 \%$ de los encuestados consideran que siempre hay una adecuada coordinación entre las áreas administrativas, el $40 \%$ dio a conocer que a veces y el $23 \%$ expreso que nunca.

El $30 \%$ de los encuestados consideran que los procesos administrativos siempre cuentan con controles que aseguran la eficacia, el $35 \%$ dio a conocer que a veces, el $23 \%$ expreso que algunas veces y el $13 \%$ expreso que nunca.

El $40 \%$ de los encuestados consideran que el cumplimiento de las metas siempre va de acuerdo a lo programado por la institución, el 33\% dio a conocer que a veces y el $28 \%$ restante expreso que no se cumplen a cabalidad dichas metas.

A raíz de los resultados obtenidos del proceso de diagnóstico se desarrolló el presente FODA de la institución.

Tabla 2: Análisis FODA

\begin{tabular}{|c|c|}
\hline Fortalezas & Oportunidades \\
\hline $\begin{array}{l}\text { - Experiencia en el negocio. } \\
\text { - Capital Privado. } \\
\text { - Talento humano profesional y con } \\
\text { experiencia. } \\
\text { - Personal Capacitado } \\
\text { - Gran variedad de productos } \\
\text { - Los productos que se ofrecen son de alta } \\
\text { calidad y con posicionamiento en el mercado } \\
\text { local. } \\
\text { - Clima laboral favorable. }\end{array}$ & $\begin{array}{l}\text { - Apoyo financiero y técnico de las entidades } \\
\text { gubernamentales. } \\
\text { - Amplio y creciente sector y mercado para } \\
\text { explotar. } \\
\text { - Políticas de Gobierno en fijación de precios } \\
\text { en la comercialización } \\
\text { - Negociación con proveedores para } \\
\text { disminución de costos. }\end{array}$ \\
\hline Debilidades & Amenazas \\
\hline $\begin{array}{l}\text { - Falta de Manual de Procedimientos y } \\
\text { organización. } \\
\text { - Carencia de Políticas en la empresa } \\
\text { - No se tiene misión y visión } \\
\text { - } \text { Inexistencia de un organigrama formal } \\
\text { - Conformismo por parte de la Alta Dirección. } \\
\text { - Desorden en la manera de realizar las tareas } \\
\text { asignadas. }\end{array}$ & $\begin{array}{l}\text { - La inestabilidad económica que puedan } \\
\text { afectar fuertemente a las Mypes. } \\
\text { - Intervención de las empresas } \\
\text { multinacionales en los distintos rubros que } \\
\text { afectan a la estabilidad de las Mypes. } \\
\text { - La alta competencia en el mercado del } \\
\text { diseño. }\end{array}$ \\
\hline
\end{tabular}

Elaborado por: Autora 


\section{Conclusiones}

Por medio del proceso de diagnóstico aplicado a la Distribuidora Telmo Macías Mora "T.M.M." se determinaron la existencia de falencias administrativas, como es la falta de Manual de Procedimientos y organización, la carencia de Políticas en la empresa, el no contar con misión y visión institucional, así como la falta de un organigrama formal hace evidente la necesidad aplicar un proceso de mejora en base a un sistema de gestión.

Puede afirmarse que, dentro de los múltiples enfoques existentes para su aplicación a la Administración en el ámbito de las organizaciones, la gestión basada en los procesos se presenta como una adecuada herramienta, que puede considerarse como fundamental para orientar a una organización hacia el logro de sus objetivos. Su aplicación genera el análisis detallado de los procesos en organizaciones de todo tipo, s, las cuales pueden modelar su forma de operación, permitiendo mejorar la gestión de cada proceso y del conjunto de procesos, para optimizar las prestaciones hacia los clientes internos y externos.

\section{Bibliografía}

Abellán, F. (2015). La gestión administrativa y su relación con los procesos de auditoria. Cuenca: Universidad de Castilla.

Alemán Carmona, A. M. (2018). El sistema de Gestión de Calidad y la atención al cliente en el Restaurante del Gourmet Urbano en el distrito de San Isidro, 2015. Lima Perú: Universidad San Martin de Porres. Obtenido http://www.repositorioacadem ico.usmp.edu.pe/bitstream/ha ndle/usmp/4339/sandoval_cfe .pdf? sequence $=3$ \&isAllowed $=$ $y$

Barragán, R. (2016). La gestión de calidad y la gestión administrativa un acercamiento al desarrollo empresarial y al fortalecimiento de las ventas. Quito: Don Bosco.

Barreiro Cedeño, I., Delgado Chávez, M., García Ponce, T., \& Mendoza Zamora, W. (2018). El control interno y su influencia en la gestión administrativa del sector público. Jipijapa: Revista Científica, Dominio de la Ciencia. Obtenido de https://dialnet.unirioja.es/desc 
arga/articulo/6656251.pdf

Coaguila Gonzales, A. F. (2017). Propuesta de implementación de un modelo de Gestión por Procesos y Calidad en la Empresa O\&C Metals S.A.C. Arequipa: Universidad Católica San Pablo. Obtenido de

http://repositorio.ucsp.edu.pe/ bitstream/UCSP/15240/1/CO AGUILA_GONZALES_ANT_ MET.pdf

Coque Arboleda, D. C. (2016). La Gestión Administrativa y su impacto en la rentabilidad de la empresa Megramicro S.A. de la ciudad de Ambato. Ambato: Universidad Técnica de Ambato. Obtenido de http://repositorio.uta.edu.ec/js pui/bitstream/123456789/228 63/1/T3604M.pdf

Dávalos Mayorga, E. R. (2016). Estructura organizacional y optimización de la gestión administrativa en la Cooperativa de Ahorro y Crédito CREDICOOP. Ambato - Ecuador: Universidad Regional Autónoma de los Andes (UNIANDES). Obtenido de http://dspace.uniandes.edu.e c/bitstream/123456789/3674/ 1/TUAMGC002-2016.pdf

Fergusson, H., \& Langford, D. (2017). Proceso de gestión administrativa en el desarrollo de las instituciones comerciales. Quito: ESPOL.

Hernández, H., \& Cardona, D. (2017). Direccionamiento Estratégico: Proyección de la Innovación Tecnológica y Gestión Administrativa en las Pequeñas

Empresas.

Barranquilla:

Direccionamiento Estratégico: Proyección de la Innovación Tecnológica y Gestión Administrativa en las Pequeñas Empresas. Obtenido de https://scielo.conicyt.cl/scielo. php?pid=S071807642017000500003\&script= sci_arttext

Lara Ocaña, J. C. (2017). Impacto de la Gestión administrativa sobre la eficiencia de la actividad pesquera artesanal Cantón Manta. Manta. Ecuador: ECASinergia. Obtenido de https://revistas.utm.edu.ec/ind ex.php/ECASinergia/article/vi ew/780/966

Lazo Cordero, K. A., \& Macas Moreno, P. R. (2018). Propuesta de mejoras en gestión administrativa en la empresa Modermueble S.A. Ciudad de Guayaquil. Guayaquil: Universidad de Guayaquil. Obtenido de http://repositorio.ug.edu.ec/bit 
stream/redug/28047/1/Propue stas-de-Mejoras-en-Gestion-

Administrativa-en-la-

empresa-Modermueble-S.A.-

Ciudad-de-Guayaqui.pdf

Mendoza Briones, A. (2017). Importancia de la gestión administrativa para la innovación de las medianas empresa comerciales en la ciudad de Manta. Manta: Universidad Laica Eloy Alfaro de Manabí, Universidad Nacional de San Marcos. Obtenido de https://dialnet.unirioja.es/servl et/articulo?codigo $=6325898$

Pitchford, P. (2017). La gestión administrativa, una estrategia para el desarrollo empresarial. Madrid España: Santa Paz.

Ramírez Casco, A. d., Ramírez Garrido, R. G., \& Calderón Moran, E. V. (2017). La gestión administrativa en el desarrollo empresarial.

Chimborazo - Ecuador: Escuela Superior Politécnica de Chimborazo, Ecuador. Obtenido de http://www.eumed.net/ce/201 7/1/gestion.html

Riveros Ortiz, K. N. (2016). Propuesta para el mejoramiento del proceso de gestión administrativa de la empresa SYSLAN SAS. Bogotá: Universidad Católica de Colombia. Obtenido de https://repository.ucatolica.ed u.co/bitstream/10983/15616/1

/Syslan\%20SAS\%20TG.pdf

Rodríguez Gómez, G. (1996). METODOLOGÍA DE LA INVESTIGACIÓN CUALITATIVA. 